\title{
Menecmi Comedy of Plautus: A Discussion between Literature and History Connected to the City of Durrës
}

\author{
Markeliana Anastasi
}

University of Tirana, Faculty of Foreign Languages, Department of Italian language Email: markeliana.anastasi@gmail.com

\author{
Doi:10.5901/ajis.2015.v4n3s1p272
}

\begin{abstract}
Menecmi is one of the best comedies of Plautus. The twin sons of Mosco and Teussimarca, originating of Syracuse, are divided into age of seven by a strange fate combination The twin brother of Syracuse is looking for land and sea his brother, and finally arrive to Epidamnos (today named Durrës) where it is settling the whole comedy. In it the author mention different elements of the city. Starting from the port, even where it is anchored navis praedatoria. Then recounting the death of his adoptive father's Menaechmus of Epidamnos the author mention that, drowned in a raging river that passes by the city. This river, which also exists today is Erzeni. Another element of the city is the forum. The presence of customs, it can be deduced from the fact that in the middle of a fight of jealousy between Menaechmus of Epidamnos and his wife, he says Portinorem domum duxi, ita omnem mihi. Another institution mentioned in the text is the prison. In the city, always from the text, it can deduce the existence of monetary circulation, as mina, sestertius, etc. Another element is the presence of different professions held in the city as: doctor, jeweler, auctioneer, porter, cook, customs officer etc. So Epidamnos is represented as a real metropolis, with its strengths and weaknesses as its twin in Greece or ancient Rome.
\end{abstract}

Keywords: Plautus, Durres, Epidamnos, forum, Erzeni river, monetary circulation, professions, the date of the comedy, port, ship, etc.

\section{La Commedia Menecmi di Plauto. Una Discussione tra Letteratura e Storia Legata alla Città di Durazzo}

Menecmi è una delle più belle commedie di Plauto. I figli gemelli di Mosco e Teussimarca, originari di Siracusa, vengono divisi in età di sette anni da una strana combinazione. II loro padre mentre partecipa in una fiera a Taranto, perde il figlio che aveva portato con se. II figlio viene trovato da un ricco mercante di Epidamno, che senza discendenza, decide di adottare il bambino perduto, portandolo nella sua città. Quando il bambino cresce, il padre adottivo lo sposa con una ragazza di buona e ricca famiglia, e quando muore, lascia tutti i suoi beni al figlio. Dall'altra parte, nella famiglia del bimbo perduto, Mosco muore proprio a Taranto dalla disperazione, e il nonno dei bambini, anche lui chiamato Menecmo ${ }^{1}$, decide di chiamare l'altro gemello Sosicle con il nome del gemello perduto. Questa decisione è la chiave di volta nello svolgimento di tutta la commedia. Il gemello di Siracusa cerca il fratello per mare e per terra, e finalmente arriva ad Epidamno dove si ambienta tutta la commedia. I gemelli con lo stesso nome e volto, vengono sottoposti a infiniti equivoci: 1-Il cuoco Cilindro confonde il Menecmo di Siracusa con il Menecmo di Epidamno, 2-Menecmo di Siracusa, pensando che il cuoco non stia bene con la testa, gli da un nummo per sacrificare un maialino contro la pazzia.3- La "buona donna" Erozia, confusa, consegna a Menecmo di Siracusa il mantello pregiato, per portarlo dal ricamatore. 4-Messinone confonde il peniculus con la spazzola delle scarpe.5- II comportamento di Erozia, viene interpretato in chiave della stupidità da parte del gemello di Siracusa.6- Spazzola, il parasita di Menecmo di Epidamno si rivolge al gemello di Siracusa come se fosse il suo padrone.7. La serva di Erozia affida il braccialetto a Menecmo di Siracusa, scambiandolo per il gemello di Epidamno.8. A Menecmo di Epidamno rientrato dal foro, gli viene chiesto di riprendere il mantello dal ricamatore, con l'accusa di averlo portato sull'ordine di Erozia.9. Spazzola rimprovera Menecmo per aver pranzato senza chiamarlo.10. Menecmo di Epidamno chiedendo a Erozia il mantello, scopre che lei glielo ha già consegnato. insieme ad un braccialetto. 11. La matrona confonde il gemello con il marito, e i suoi insulti vengono ricambiati con delle offese ancor più gravi. 12. II gemello di Siracusa minaccia il vecchio di tagliarlo in due con la scure o investirlo con la quadriga.13. L'incontro con il medico del gemello di Siracusa.14. L'incontro con il medico di Menecmo di Epidamno15. Messinone e

${ }^{1}$ Forse era un nome in voga in quell'epoca, essendo che anche uno dei più importanti matematici e studiosi di geometria dell'epoca, si chiamava Menecmo vissuto tra 380 a.C. circa - 320 a.C. circa, in Tracia. 
Menecmo di Epidamno, la difesa e la liberazione.

Tutti i quindici equivoci ingarbugliano così tanto la vicenda che ci vuole un deus ex macchina per distendere le anime ferventi. Esso si raggiunge tramite la conferma di diversi elementi che distinguono i nostri personaggi. I due Menecmi trovandosi di fronte, si guardano increduli, vogliono con tutto il cuore che quello che pensano sia vero, ma per questo devono avere delle conferme. In loro aiuto viene Messinone che domandando su fatti sconosciuti, porta lo svolgimento della commedia verso il lieto fine. Messinone di Epidamno viene riconosciuto dal fatto che è figlio di Mosco, nato a Siracusa, diviso dal padre e portato in Epidamno in età di sette anni e, ne aveva un fratello gemello La rivelazione del nome del fratello, Sosicle conferma l'identità di Menecmo di Epidamno, invece l'identificazione di Menecmo di Siracusa viene tramite la conferma del nome della madre, Teusssimarca.

Se il gioco degli equivoci è l'asse portante della comicità, quello dell'indovinello porta alla gioia, e trasmette entusiasmo. Tutti questi elementi fanno che questa commedia sia una delle più belle mai scritte nella storia della letteratura. Questo fatto inconfutabile si conferma grazie alla sua rielaborazione da diversi autori nel corso dei secoli. La più interessante è La commedia degli errori di William Shakespeare?2.

Una commedia molto di moda durante il '500 in età umanistica, oggetto di lavoro filologico, come anche prescelta in occasioni straordinari come: nel 1486 alla corte di Ercole I in onore di Francesco II Gonzaga, promesso sposo a Isabella d'Este; nel 1488 per Lorenzo il Magnifico. II Prologo venne recitato da Angelo Poliziano; nel 1488 per Francesco II Gonzaga e Ludovico il Moro.

Si è parlato tanto per quanto riguarda l'interpretazione plautina nella commedia Menecmi, della gente di Epidamno. Addirittura si è arrivato ad abusare con l'uso dei versi della commedia, a discapito degli onesti cittadini di questa bellissima città. Questo fatto è un motivo in più per chiarire i versi di Paluto. In essi si parla di una certa categoria di persone. Generalmente sono i cosi detti angiport . Imbroglioni, ladri, meretrici della peggior specie. Una rappresentazione letteraria creata proprio per costruire quel gioco di equivoci, che ti porta alla comicità per eccellenza. Nella letteratura in generale e nel genere comico in particolare, questi personaggi o si creano d'immaginazione, o si basano su fatti storici. Però in un'opera letteraria la cosa più importante è proprio l'arte dello scrivere bene, nella commedia particolarmente, la scelta di un certo tipo di carattere, mettendolo in condizioni sui generis, è il gioco dell'autore che porta ilarità in scena. Questo è l'elemento base delle commedie di Plauto. Sicuramente anche l'autore medesimo non abbaia avuto l'intenzione di alterare l'immagine di una intera città. Le affermazioni di Plauto si connettono con il gioco degli equivoci, molto caro all'autore. Dicendo che in un posto d'arrivo, ignoto per te, ti succederà qualche cosa di brutto, sicuramente che ti prepari per il peggio. La reazione difensiva dei personaggi, crea quell'equivoco, che naturalmente è la base della comicità palutina.

Si dice che in questa città non esci senza danno. Ma di fatto qual è il danno che subisce Menecmo di Siracusa? È stato servito, e riverito, e alla fine, è lui che ruba il mantello e il braccialetto d'oro a Erozia: Dille che io penso a tutto... (sottovoce) che penserò a vendere tutto al miglior prezzo che si può spuntare. E il suo servo Messinone? Cosa li succede di brutto in questa città? Niente addirittura diventa un uomo libero.

Per quanto riguarda l'argomento donne, ne abbiamo di fronte due tipologie: la prima è Erozia; in quasi tutte le commedie di Plauto possiamo incontrala una, sia di Epidamno, di Roma3 ${ }^{3}$ Grecia ecc. Dall'altra parte c'è la figura della moglie di Menecmo di Epidamno, Potrebbe essere gelosa del suo marito, ma lo accusa senza motivo? Menecmo frequenta Erozia, ruba alla moglie mantelli e gioielli per darla a lei. Chi è quella donna che non manifesterebbe segni di gelosia. Non solo che viene ripudiata e rubata, ma alla fine viene anche messa in asta per essere venduta da parte del marito infedele.

Lo stesso argomento viene rielaborato da Trissino nella commedia Simillimi e l'ambientazione dei personaggi è a Palermo ${ }^{4}$. Forse si deve parlare male generalmente di tutti gli abitanti di questa città, per via di una commedia?!...

Per concludere il filo del discorso: Epidamno uguale danno, vorrei sottolineare che, fin che siamo nel campo della commedia possiamo anche ridere, ma se i limiti si calpestano il prodotto creato non potrebbe più essere considerato letteratura.

\footnotetext{
${ }^{2}$ Anche altri autori hanno rielaborato la commedia come: Trissino, Bibbiena, Jean Rotrou, Jean François Regnard, e ancora La dodicesima notte di William Shakespeare.

${ }^{3}$ Bacchide I, e II nella commedia Bacchidi con ambientazione ad Atene, Filocomasia nella commedia Miles gloriosus con ambientazione ad Efeso, Flilènia nella comemdia Asinara, Planesio in Curculio, la Cortegiana in Epidicus Filemazia in Mostellaria, Lemniselene in Persa; Fenicia in Pseudolus; Fronesio in Truculentus;ecc.

${ }^{4}$ Trissino, G. G., I Simillimi, editore Daelli 1864.
} 


\section{Elementi della Città}

La città descritta da Plauto nella commedia Menecmi è Epidamno, odierna Durazzo. In essa si menzionano elementi diversi della città. Iniziando dal porto, dove addirittura è ancorata la navis praedatoria, o navis peregrina. Sono queste le parole del servo di Menecmo di Siracusa, Messinone. Poi raccontando la morte del padre adottivo del Menecmo di Epidamno si menziona che, muore annegato in un fiume impetuoso che passa nelle vicinanze della città. Questo fiume, esistente anche nei nostri giorni è Erzeni, fluvium rapidum, ab urbe haud longule, che scorre non lontano dalla città di Durazzo ${ }^{5}$. In un'iscrizione trovata a Durazzo si dice che l'acquedotto costruito dall'Imperatore Adriano (117-138 d.C), prendeva acqua dal fiume Erzen ${ }^{6}$, Questo acquedotto forniva con acqua pulita le fontane pubbliche della città, le terme e qualche casa di personaggi importanti e ricchi. L'acquedotto di Dyrracchium prende l'acqua potabile dal fiume Ululeus ${ }^{7}$ (odierno fiume Erzen) distante $20 \mathrm{~km}$ dalla città. Fino all'anno 1958 l'approvvigionamento con i rifornimenti idrici della città di Durazzo si copriva proprio dalle acque di questo fiume. Poi ha fornito parzialmente diversi quartieri periferici della città come, la zona industriale di Shkozet e l'intera zona balneare chiamata Plazh, praticamente il quartiere 13 e 14 fino all'anno 1975, quando si costruisce l'impianto idrico di Fushë Kuqe.

Un altro elemento della città è il foro. Menecmo di Epidamno racconta che è andato al foro, per partecipare in un assemblea per difendere un cliente nei confronti del popolo, edili, patroni, e magistrati. 587- aut ád populum aut ín iure aut ápud aedilem rés est. Contro di esso sono tre testimoni accaniti che lo accusano in modo fervente. 595- omnibus male factis testes tres aderant acerrum.

La presenza della dogana, si deduce dal fatto che in mezzo ad una lite di gelosia fra Menecmo di Epidamno e la moglie, egli dice: 117 Portitorem ${ }^{8}$ domum duxi, ita omnem mihi. Un'altra istituzione menzionata nel testo è il carcere. Menecmo di Epidamno in mezzo ad una forte discussione con lo suocero gli dice che: 942- et ob eam rem in carcerem. In questo testo abbiamo notizia anche di forme di punizione come ad esempio la tortura con la forca ${ }^{9}$. Nello stesso dialogo con lo suocero, il protagonista dice: 944- caesum virgis sub furca scio.

Nella città, sempre dal testo, si deduce l'esistenza della circolazione monetaria. Si menziona la mina, quando Menecmo di Epidamno racconta il prezzo pagato per il mantello della moglie: 205- Quattuor minis ego emi istanc anno uxori meae. pen. Quattuor minae perierunt plane, ut ratio redditur. Nell'antico sistema monetario greco un talento era uguale a sessanta mine, e una mina era uguale a 100 dracme. Un'altra notizia su questo tipo di moneta si riscontra nell'opera "Apologia di Socrate" di Platone ${ }^{10}$. Anche la circolazione del sesterzio, è presente nel testo. Esso era anche usato come unità di conto standard, rappresentato nelle iscrizioni con il monogramma HS. Somme elevate erano registrate come sestertia milia, migliaia di sesterzi, con la parola milia spesso omessa e sottintesa. Ė difficile fare comparazioni con le valute o i prezzi attuali, ma per la maggior parte del I secolo un legionario ordinario era pagato 900 sesterzi per anno, che salirono a 1200 sotto Domiziano (81-96), equivalenti a 3,3 sesterzi al giorno.

Se facessimo un parallelo tra asse e euro, sulla base del prezzo corrente del pane, che si aggira, in media, intorno a 3 euro al chilo, potremmo affermare che un asse sarebbe equivalso a 1,5 euro; un sesterzio a 6 euro'11. Nel testo, Messinone nel ruolo di banditore d'asta, 1153 praeconium mi ut detis, menziona che: Vix credo tota auctione capiet quinquagesies. Nunc; dunque il calcolo che la vendita sfiorerà, tutto sommato, i cinque milioni di sesterzi. Non sappiamo con certezza come funzionava l'economia dell'epoca, ma se facessimo due conti: $5000000 \times 6 €=30000000$ di euro. La cifra guadagnata da parte di Menecmo di Epidamno, è importante per l'epoca in cui le vicende plautine si svolgono. Naturalmente tutti questi calcoli si devono prendere con molta criticità e equilibrio. Un'altra moneta menzionata è il nummo. In un momento della commedia Erozia viene ordinata da Menecmo di preparare il pranzo. Lei chiama il suo cuoco Cilindro e li dice: 218 sportulam cape atque argentum. eccos tris nummos habes. Poi nel dialogo fra Cilindro e

\footnotetext{
${ }^{5}$ Nella prima foto si rappresenta il fiume Erzen, e nella seconda anche la mappa dove si vede benissimo la vicinanza del fiume con la città di Durazzo.

${ }^{6}$ Miraj. L., rivista "lliria" 1991, p. 250-251.

${ }^{7}$ Miraj, F.,- Myrto, H., Ujësjellësi i Durrësit, Rivista “lliria”, 1982, 1, p. 131-156.

${ }^{8}$ Portitorem, il doganiere, come la moglie, fa molte domande, per la metafora, cf. Asinara. 159, 241 e Cicerone. Ad Atticum. 2,16,4.

${ }^{9} \mathrm{La}$ più crudele delle torture era la forca dell'eretico, uno strumento che veniva conficcato nello sterno e sotto il mento, con le estremità acuminate, cosi da bloccare all'accusata ogni movimento, permettendole solo di sussurrare le proprie confessioni

10 Platone, Apologia del Socrate, pag.13, XXVIII. racconta come dopo la condanna del filosofo da parte dei giudici ateniesi, questi si sarebbe potuto auto-comminare un'ammenda pari ad 1 mina d'argento, o al massimo 30 mine con la garanzia degli allievi a lui più vicini. Tale pena non avrebbe certo cagionato alcun danno a Socrate in quanto filosofo.

11 II Faro, Quotidiano telematico del Mediterraneo, Quanto valeva una moneta romana, e cosa era possibile acquistare. 17-09-2012.
} 
Menecmo di Siracusa, il secondo dice: 288. quibus hic pretiis porci veneunt sacres sinceri? cyl. Nummis. men. Nummum a me accipe. II nummo viene menzionato anche nel dialogo fra L'ancilla di Erozia e Menecmo di Siracusa 541inauris da mihi faciendas pondo duom nummum, stalagmia. Non sappiamo con esattezza per quale tipologia di nummo si parla. Nella traduzione albanese il traduttore usa il termine dracma ${ }^{12}$. Un'altra moneta menzionata è l'oncia. Mentre la serva di Erozia chiede a Menecmo di Siracusa di portare dall'orefice il braccialetto di Erozia, gli dice di aggiungere un oncia d'oro: 526-atque huc ut addas auri pondo únciam.

Alla fine il Menecemo di Epidamno chiede aiuto ai cittadini della sua città 1000- Epidamnienses, subvenite, cives. quin me mittitis? Questo è un fatto inconfutabile che si parla della civiltà di Epidamno. Non puoi chiedere aiuto dove non lo aspetti. La vicenda si svolge per le vie della città, 1005- Epidamnii cives, erum meum hic in pacato oppido luci deripier in via, dice Messinone; nelle quali come mezzi di trasporto circolavano le quadrighe. Questo fatto viene menzionato nel dialogo tra lo suocero, il Senex (il vecchio) e Menecmo di Epidamno e 934- etiam me iunctis quadrigis, parola che si ripete diverse volte nella commedia. Sicuramente nelle vicinanze della città si allevavano cavalli, proprio per l'uso sia di comunicazione ma anche come animali da soma ecc.

Un'altra istituzione menzionata nella commedia è la vendita all'asta. ${ }^{13}$

Da un'altra angolazione nella commedia si presentano le professioni della gente di Epidamno. Una delle figure più interessanti è quella del medico. La sua professionalià viene messa in evidenza da diversi elementi nella commedia. II vecchio quando parla della bravura del medico dice che: 885 ait se óbligasse crus fractum Aesculapio/ Apollini autem brachium. nunc cogito,/ utrum me dicam ducere medicum an fabrum; o quando il medico tranquillizza il vecchio della sua capacità dicendo che, egli cura seicento persone al giorno 896 Quin suspirabo plus sescenta ín die ; Poi quando il medico chiede sullo stato fisico del malato 890-num laruatust aut cerritus? fac sciam/num eum veternus aut aqua intercus tenet? Dunque parla di diversi sintomi di malattie come quelle mentali, l'idropsia ${ }^{14}$, l'isterismo ecc.

Un momento molto importante è quando nella commedia si parla dell'elleborio, una pianta menzionata da Plinio il Vecchio nella sua Naturalis historia si dilunga alquanto nel descrivere pregi e inconvenienti dell'elleboro bianco e nero. Un caso interessante è la cura contro epilessia del tribuno Marco Livio Druso che si era recato ad Anticira dove si liberò delle crisi epilettiche. Questo nome è presente sia nel Vocabolario dalla lingua albanese ${ }^{15}$, sia nel libro dell'uso delle piante omeopatiche ${ }^{16}$ in familia. Una pianta che si usa per curare i problemi neurologici. Infatti nell'opera, il medico propone una cura con elleboro, in albanese shpendër ${ }^{17}$ ose lule taçe, per venti giorni filati 950- elleborum potabis faxo áliquos viginti dies, dunque la commedia ci aiuta anche con una ricetta come curarsi con l'elleborio in Epidamno. L'uso del anamnesi ${ }^{18}$ per creare comicità è un'altra strategia di Paluto quando chiede sul tipo di vino scelto da Menecmo, lo stato fisico degli occhi, sull'intestino, il sonno ecc. L'ilarità si crea tramite la serietà con cui lavora il medico che addirittura sottolinea che sta usando l'anamnesi 922- mane modo, etiam percontabor alia; e le risposte quasi innocenti di

\footnotetext{
12 Laçaj, H., Plauti, Menehmët,N.Sh. Botimeve "Naim Frashëri", Tiranë 1959.

${ }^{13}$ Donadio, N., La auctiones private all'epoca di Plauto: consuetudini, regole, pratiche nelle vendite all'asta nel mondo romano e loro tracce nella "palliata" latina, LED 2007. era un istituto utilizzato nel mondo antico sia in Grecia e nel Egitto tolemaico, sia nell'Italia preromana che a Roma. Le più antiche furono verosimilmente le auticiones pubbliche testimoniate già alla fine del V secolo13. La figura del praeco praeconium e alla relativa funzione nel procedimento delle vendite all'asta, si accenna in:1154, Praeconium mibi ut detis. Infine di auctio in genere sia in riferimento a vendite pubbliche che private si parla nei seguenti versi: 1152Auctionem bis faciam et vendam quicquid est: 1157Auctio Menaechmi mane sane septi; 1161Vix credo auctione tota capiet quinquagesis aesMenecmo di Epidamno in vista all'rientro nella terra d'origine a Siracusa decide di mettere in asta si sui beni, addirittura la moglie. A questo scopo, su offerta di Messinone medesimo da incarico al ex schiavo di fare da banditore all'asta. Segue quindi la rappresentazione di Messionone nell'atto di procedere alla proclamazione dell'auctio con indicazione della data, l'ora, il luogo di svolgimento della vendita, degli oggetti messi all'asta. L'auctio conclamari plautino si connette direttamente con la circostanza che le vendite all'asta appaiono disposte per esigenze straordinarie e siano destinate a concludersi in breve tempo. L'auctio dei beni di Menechmo di Epidamno, annunciato a brevissima distanza nel lasso di tempo di sette giorni, dal momento della proclamazione dell'avviso d'asta, per consentire ai due gemelli di lasciare in minor tempo possibile Epidamno per la volta di Siracusa. Non necessariamente le vendite all'asta si svolgessero al forum. Non si può escludere che nelle città con uno sbocco sul mare, le aste si tenessero anche al portus, in prossimità degli attracchi delle navi cariche di merci provenienti d'oltremare. o atria actionaria In queste ipotesi la proclamazione della vendita dovesse avvenire in forma orale.

${ }^{14}$ Le cronache antiche fanno riferimento alla malattia in numerosissimi casi e testimoniano che questa condizione era spesso mortale.

${ }^{15}$ Fjalor I gjuhës së sotme shqipe, Tirane 1980, pag. 1962.

${ }^{16}$ Kokalari, P., Sima, Z, Xinxo P., Bimët mjekësore në familje, botim I drejtorisë së arsimit shëndetësor, Tiranë 1980, pag. 402.

17. Plauti, Menehmët,N.Sh. Botimeve "Naim Frashëri", Tiranë 1959, pag. 106, v. 1369.

${ }^{18}$ Raccolta a scopo diagnostico di tutte le notizie riguardanti i precedenti fisiologici e patologici, personali ed ereditari, di un paziente.
} 
Menecmo. 913. album an atrum(nero) vinum potas? 923- solent tibi umquam óculi duri fieri? 925- en umquam intestina tibi crepant, quod sentias? 932- perdormiscin usque ad lucem? facilen tu dormis cubans?

Altri professioni menzionati nell'opera sono quelli dell'orefice: 525 - ut hoc una opera <sibi> ad aurificem deferas,; il ricamatore469- Pallam ad phrygionem fert confecto prandio; il cuoco, il portiere 671- hic est ianitor?, il cocchiere Eu edepol ne tu, ut ego opinor, esses agitator probus, il doganiere 117 Portinorem domum duxi, ita omnem mihi, edile aedilem rés est, magistrato, patrono 581- sollícitos patrónos habént.ecc.

II modo di vestirsi ad Epidamno viene fuori proprio da un elemento centrale della commedia, il mantello. Menecmo compra alla moglie un mantello con valore di quattro mine, e dopo un anno decide di regalarla alla sua bella Erozia. Dunque le donne della città come soprabito vestivano il mantello, indossavano vestiti di lana, abiti in porpora 120 lanam, aurum vestem, purpuram bene praebeo nec quicquam eges, mentre gli uomini vestivano clamide e mantello. Per quanto riguarda i gioielli, nel testo si menzionano le armille 536- Vbi illae armillaé sunt, quas una dedi?, il braccialetto 527 iubeasque spinter novom reconcinnarier., gli orecchini pendenti 542 faciendas pondo duom nummum, stalagmiaut te libenter videam, quom ad nos veneris ecc.

Per quanto riguarda l'arredamento delle case, l'unica testimonianza è la parola armadio. Quando Menecmo di Siracusa parla con la serva di Erozia, lei li racconta che il braccialetto che lui ha donato alla sua bella l'avrebbe rubato dall'armadio della moglie 530 Hoc est quod olim clanculum ex armario.

\section{II Mondo Culinario di Epidamno nella Commedia Menecmi}

Nella commedia di Plauto troviamo indizi del mondo culinario della città. Spazzola quando parla di Menecmo racconta di cene organizzate degne di Cerere 101 cerialis cenas dat con montagne di piatti. Già nell'atto primo Menecmo di Epidamno invita la sua bella ti preparare un bel pranzo. I piatti menzionati sono: Animelle di porco, prosciutto, testa di maiale 210 glandionidam suillam, laridum pernonidam, aut sincipitamenta porcina aut aliquid ad eum modum. In Albania anche oggi si preparano questi cibi, in particolare un tipo di prosciutto, viene preparato con carne suina sotto sale, appesa e conservata in un luogo asciutto e buio, chiamata Pastërmà.

La presenza del mercato, si conferma nelle parole di Menecmo di Epidamno quando chiede di mandare al mercato Cilindo per fare la spessa per il pranzo e dalla lista della spesa si deduce che al mercato civico potevi comprare carne, prosciutto, testa di maiale ecc.

In città esistevano delle locande 1035- nunc ibo in tabernam, vasa atque argentum tibi, dove le persone potevano mangiare e alloggiare.

Plauto sostiene che Epidamno è un posto dove si beve molto. Questo fatto lo conferma Menecmo quando decide di organizzare un pranzo con Erozia e di bere tanto 187 In eo uterque proelio potabimus; uter ibi melior bellator erit inventus cantharo. La tipologia della bevanda esce fuori nel dialogo fra il medico e Menecmo quando li chiede se il vino lo preferisce bianco o nero 913.album an atrum vinum potas? Dunque è il vino la bevanda che si consuma ad Epidamno. Questo è un fatto inconfutabile essendo che le scoperte archeologiche sia in entroterra che al mare di Durazzo hanno evidenziato una vasta attività di trasporto vinario, addirittura sono rinvenuti nella città elementi di atelier dove si producevano le anfore ${ }^{19}$.

Un altro aspetto interessante della città, si raffigura nella commedia nelle superstizioni e credenze popolari. II primo riguarda il sacrificio contro la pazzia, che consisteva nel sacrificare un maialino, che addirittura costava un nummo; il secondo riguarda quando il cuoco Cilindo pensa che Menecmo è pazzo che si getta addosso il malocchio Insanit hic quidem, qui ipse male dicit sibi.

\section{La Data}

L'opera di Plauto I Menecmi si pensa essere stata scritta alla fine del terzo secolo a. ${ }^{20}$. Se Plauto fosse stato molto fedele alla storia, e dall'altra parte se per navis praedatoria, ancorata al porto di Epidamno, si presume una nave di pirati illirici, possiamo stringere un po la data storica. Parliamo si per la seconda metà del terzo secolo (da 250-200 a.C) ma se

19 Toçi, V. Popullsia ilire e Dyrrahut në dritën e të dhënave historike-arkeologjike, p. 382, in Kuvendi i studimeve ilire Tiranë 15-20 settembre 1972. ; La pubblicazione degli Atti del Convegno dell' Accademia delle Scienze dell'Albania. Shtypshkronja "Mihal Duri", Tiranë 1974.

20 Bessone, L., Scuderi, R., Manuale di storia romana, Monduzzi editore, Bologna 1994, pag 117-118. 
consideriamo le condizioni romane imposte alla regina Teuta al 229 a.C. ${ }^{21}$, possiamo collocare cronologicamente il trama prima del $229 \mathrm{a}$. C il che significa che la data più probabile potrebbe essere considerata il decennio 240-230 a.C. Ma se la data di nascita di Plauto fosse il 255 a.C., e la sua fioritura letteraria si colloca verso il 200 a.C., allora i fatti accaduti, possono essere svolti fra la seconda e la terza guerra illirica. In un passo della commedia, Erozia menziona che Menecmo è figlio di Mosco nato Siracusa dove ha regnato Agathocles, Phintia, il terzo Liparo che lasciò il trono a Gerone che tuttora lo tiene 409. ubi rex Agathocles regnator fuit et iterum Phintia, tertium Liparo, qui in morte regnum Hieroni tradidit,nunc Hiero est? Si tratta di Gerone II, vissuto tra 270-216 a.C. Da questo dato importantissimo possiamo avanzare l'ipotesi che l'opera Menecmi sia scritta prima del 216 a.C. dunque Plauto scrive la commedia in un età di circa 35-40 anni.

\section{Conclusioni}

La commedia di Paluto Menecmi si svolge nella città di Epidamno, odierna Durazzo. Tramite le parole dell'autore si crea l'immagine della città, la quale combaccia anche con le fonti storiche letterarie. In questa ricerca si è cercato di fare una ricostruzione fedele di Epidamno dalla quale risulta una metropoli simile a Roma e Atene, con il suo forum, il suo mercato, il porto, la dogana, il carcere ecc. Nella città si svolgono professioni diversi come giudice, medico, orafo, cuoco, in funzione ad una società elevata e simile alle sue coetanee nel Mediterraneo. Un mondo di padroni e sciavi, di pirati, di spose e amanti. In particolare si deduce la cucina del posto, il modo di vivere e vestire, la circolazione monetaria, le aste del posto. Naturalmente tutta questa informazione non è solo importante per la ricostruzione storica e letteraria del posto, ma può spingerci a pensare (basandosi proprio nella descrizione dettagliata del posto) che Plauto medesimo sia stato a Epidamno, e abbia parlato di una città dove ha vissuto per un piccolo periodo di tempo.

\section{References}

Bessone, L., Scuderi, R., Manuale di storia romana, Monduzzi editore, Bologna 1994.

Cabanes, P., Les Illiyriens de Bardylis à Genthios, Paris 1988.

Ceka, H., Monedhat e Durrësit dhe të Apollonisë, Tiranë 2008.

Donadio, N., La auctiones private all'epoca di Plauto: consuetudini, regole, pratiche nelle vendite all'asta nel mondo romano e loro tracce nella "palliata" latina, LED 2007.

Fjalor I gjuhës së sotme shqipe, Tirane 1980.

Frashëri, K., Monedha, krediti dhe banka në Shqipëri gjatë shekujve, Toena, Tiranë 2011.

Giacchero M., Storiografia romana antica, Bozzi editore, Genova 1973.

Kokalari, P., Sima, Z, Xinxo P., Bimët mjekësore në familje, botim I drejtorisë së arsimit shëndetësor , Tiranë 1980

Laçaj, H., Plauti, Menehmët,N.Sh. Botimeve "Naim Frashëri", Tiranë 1959

Miraj, F.,- Myrto, H., Ujësjellësi i Durrësit, Rivista "Iliria", 1982, 1

Miraj, L., Rivista "lliria" 1991.

Toçi, V. Popullsia ilire e Dyrrahut në dritën e të dhënave historike-arkeologjike, p. 382, in Kuvendi i studimeve ilire Tiranë 15-20 settembre 1972. ; La pubblicazione degli Atti del Convegno dell'Accademia delle Scienze dell'Albania. Shtypshkronja "Mihal Duri", Tiranë 1974.

Trissino, G. G., I Simillimi, editore Daelli 1864.

Plauto, I Menecmi, Rizzoli 1984, con testo latino di fronte.

Polibio, Storie, libro I-II, a cura di Domenico Musti, edizione BUR, Milano 2001.

11821Polibio, Storie, libro I-II, a cura di Domenico Musti, edizione BUR, Milano 2001, libro secondo, pag 431-433. Esse si svolgono prima per debellare la pirateria e successivamente per la conquista della regione. La prima Guerra Illirica svolta con 20.000 fanti, 2.000 cavalieri e 200 navi capeggiata dai consoli Lucio Postumio Albino e Gneo Fulvio Centumalo e da parte di Illiri regina Teuta e Demetrio di Faro, si conclude a discapito degli Illiri con la conseguenza di impegnarsi ad assicurare che nessuna nave da guerra illirica e non più di due mercantili si spingessero oltre Lisso. 